\title{
Efficacy of Naoxueshu in acute spontaneous intracerebral hemorrhage: a multicenter observational study
}

\author{
Juexian Song ${ }^{1} \cdot{\text { Yuting } \mathrm{Nie}^{1} \cdot \text { Xinzuo Qin }}^{1} \cdot$ Pingping Wang ${ }^{1} \cdot$ Huiqiang $\mathrm{Lu}^{2} \cdot \mathrm{Li} \mathrm{GaO}^{1} \mathbb{C}$
}

Received: 12 April 2021 / Accepted: 26 August 2021 / Published online: 16 September 2021

(c) The Author(s) 2021

\begin{abstract}
Objective To evaluate the efficacy and safety outcome and related risk factors of Naoxueshu in the treatment of acute SICH. Methods Two hundred twenty patients were enrolled in this study. Diagnosis of SICH was based on neuroimaging. All the patients received regular treatment and Naoxueshu oral liquid $10 \mathrm{ml} 3$ times a day for 14 consecutive days. Surgical intervention was conducted as needed. Efficacy and safety outcomes were evaluated.

Results Hematoma volume decreased significantly 7 days after Naoxueshu treatment (from $27.3 \pm 20.0$ to $15.1 \pm 15.1 \mathrm{ml}$, $P<0.0001)$, and it decreased further in 14-day result $(6.9 \pm 10.4 \mathrm{ml}, P<0.0001)$. Patients' neurological function was improved remarkably with NIHSS scores from baseline 13 points to 7 -day 7 points $(P<0.0001)$ and 14 -day 4 points $(P<0.0001)$. Cerebral edema was relieved only 14 days after Naoxueshu treatment (from 3 to 2 points, $P<0.0001$ ). No clinically significant change was found in 7-day and 14-day safety results. Female sex was related independently to large 7-day hematoma volume and worse 7-day NIHSS score while it would not affect patients' 14 -day outcomes. Rare cause of SICH $(B=17.4$, $P=0.009)$ alone was related to large 14-day hematoma volume. Worse baseline NIHSS score $(B=0.3, P=0.003)$ and early use of Naoxueshu $(B=2.9, P=0.005)$ were related to worse 7-day and14-day neurological function.

Conclusion Naoxueshu oral liquid could relieve hematoma volume and cerebral edema safely; meanwhile, it could improve patients' neurological function. Sex, cause of SICH, and time from onset to receive Naoxueshu should be taken into consideration in the treatment of SICH.
\end{abstract}

Keywords Intracerebral hemorrhage $\cdot$ Hematoma $\cdot$ Traditional Chinese medicine $\cdot$ Naoxueshu $\cdot$ SICH

\section{Introduction}

Intracerebral hemorrhage (ICH) accounts for approximately $10 \%$ of all strokes [1]. Spontaneous intracerebral hematoma (SICH) is defined as nontraumatic bleeding into the brain parenchyma with abrupt onset of severe headache, altered level of consciousness, and/or focal neurologic deficit [2]. On neuroimaging, we can find focal collection of blood within the parenchyma. SICH is characterized by high mortality and morbidity rate that 30-day mortality has been reported to be approximately $40-50 \%$ [3, 4]. SICH has become one of the heavy burdens to the society and the

Li Gao

Xuanwugaoli@126.com

1 Department of Neurology, Xuanwu Hospital, Capital Medical University, Beijing 100053, China

2 The Key Laboratory of Development Biology, College of Life Sciences, Jinggangshan University, Ji' an, China family. However, until today, we have limited treatments for SICH because the mechanism of SICH is not clear. According to the latest AHA/ASA guidelines, reversal of coagulopathy and control of hypertension remain the main therapy of SICH [5].

Finding out an effective neuroprotective drug has been on the schedule of neurologists around the world for many years. Unfortunately, only limited success has been reported in preclinical and clinical trials evaluating numerous compounds [6]. On the other hand, traditional Chinese medicine (TCM) is very popular in all developing countries. According to a $2003 \mathrm{WHO}$ report, it accounts for 30 to $50 \%$ of the total medicinal consumption in China [7]. TCM has been used for the treatment of stroke for thousands of years in China. Naoxueshu oral liquid, a TCM patent drug including leech, astragalus root, Rhizome Chuanxiong calamus, and Achyranthes, is designed on the concept of TCM theory that "Qi is the commander of blood; blood is the mother of Qi" [8]. Many small sample studies have demonstrated 
that Naoxueshu can replenish Qi, activate blood circulation, and remove blood stasis, thus relieving symptoms of SICH [9-13]. The aim of this study was to evaluate the efficacy of Naoxueshu in the treatment of acute SICH in a relatively large-scale population in China. Moreover, we analyzed risk factors that could affect patients' outcomes when they took Naoxueshu into consideration.

\section{Material and methods}

We prospectively enrolled the patients diagnosed with SICH between December 2016 and August 2019 in 6 hospitals in Beijing, China. Diagnosis of SICH was according to the 2015 AHA/ASA SICH guidelines [5], and was confirmed by magnetic resonance imaging (MRI) or computed tomography (CT). The Ethics Committee of Xuanwu Hospital of Capital Medical University approved this study. Data of all patients were prospectively collected and retrospectively analyzed. The procedures were performed with the patient hospitalized and the written consent of the patients was obtained before the procedure.

\section{Intervention}

After the admission, patients were randomized to Naoxueshu group and Control group. This was an addition on design study. All the patients in two groups received standard of care which include $20 \%$ mannitol of dehydration treatment, general monitoring and nursing care, blood pressure control, and supportive therapy. Naoxueshu oral liquid $10 \mathrm{ml}$ 3 times a day was given to the patients for 14 consecutive days in Naoxueshu group. Surgery including craniotomy, decompressive craniectomy, and minimally invasive surgical evacuation was conducted if deteriorating vital signs or neurological function was found in both groups.

\section{Main outcomes}

Efficacy outcomes were hematoma volumes, National Institutes of Health Stroke Scale (NIHSS) scores, and cerebral edema scores of 7 days and 14 days after Naoxueshu treatment. Safety outcomes were 7-day and 14-day changes in blood routine examination, coagulation function, liver function, and renal function. The NIHSS rater was an independent investigator who was blinded of our study in each site. Hematoma volume $(\mathrm{ml})=\pi / 6 \times$ length $(\mathrm{cm}) \times$ width $(\mathrm{cm}) \times$ high $(\mathrm{cm})$ [14], and it was determined by CT scanning (Siemens 64-slice CT machine, section thickness, $5 \mathrm{~mm}$; gap, $5 \mathrm{~mm}$; pitch, 1; tube current, $304 \mathrm{~mA}$; and voltage, $120 \mathrm{kV}$ ).

The CT results were evaluated by an independent radiologist who was blinded in our study with the following protocol, cerebral edema severity was evaluated by a 4-grade scale: (1) No cerebral edema; (2) formation of perihematoma edema zone; (3) manifestation of grade 2 plus ventricle compression; (4) midline shift in addition to manifestation of grade 2 and grade 3 .

\section{Data analysis}

All statistical analyses were conducted by SPSS 19.0 (SPSS Inc., Chicago, IL, USA). Continuous variables were presented as means \pm standard deviations $(\mathrm{SD})$ if they were normally distributed; if not they were presented as median (interquartile range). The category variables were presented as numbers and frequencies. Outcome comparison was done by repeated measures ANOVAs and all the results of repeated measures ANOVAs were corrected with the Greenhouse-Geisser correction. Post hoc Bonferroni's test was performed for pairwise comparisons. Risk factors related to efficacy outcomes were analyzed by linear regression. A $P$ value less than 0.05 was considered statistically significant.

\section{Results}

\section{Baseline characteristics}

The baseline characteristics of the patients are shown in Table 1. Between December 2016 and August 2019, 220 patients were enrolled in this study. SICH patients were $55.8 \pm 11.9$ years with male predominant. Vital signs were normal except that both systolic pressure $(159.3 \pm 25.7 \mathrm{mmHg})$ and diastolic pressure $(96.5 \pm 18.1 \mathrm{mmHg})$ were elevated. Among them, $69.5 \%$

Table 1 Baseline characteristics of the patients

\begin{tabular}{ll}
\hline Characteristics & \\
\hline Age (years) & $55.8 \pm 11.9$ \\
Male sex & $143(65.0 \%)$ \\
Heart rate $(n / \mathrm{min}$, median, IQR) & $80(71-87)$ \\
Respiratory rate $(n / \mathrm{min}$, median, IQR) & $19(18-20)$ \\
Temperature $\left({ }^{\circ} \mathrm{C}\right)$ & $36.7 \pm 0.5$ \\
Systolic pressure $(\mathrm{mmHg})$ & $159.3 \pm 25.7$ \\
Diastolic pressure $(\mathrm{mmHg})$ & $96.5 \pm 18.1$ \\
Cause of SICH & \\
Hypertension & $153(69.6 \%)$ \\
Intracranial vessel malformation & $61(27.7 \%)$ \\
Others & $6(2.7 \%)$ \\
Time from onset to receive NXS (days) & $1.7 \pm 0.8$ \\
\hline
\end{tabular}

Notes: IQR, interquartile range; $S I C H$, spontaneous intracerebral hemorrhage; NIHSS, National Institutes of Health Stroke Scale; NXS, Naoxueshu oral liquid 
were hypertensive $\mathrm{ICH}$, and $27.7 \%$ were caused by intracranial vessel malformation. Average time from SICH symptom onset to receive Naoxueshu oral liquid was $1.7 \pm 0.8$ days.

\section{Outcomes comparison}

Efficacy outcomes are showed in Table 2. Hematoma volume decreased significantly 7 days after Naoxueshu treatment (from $27.3 \pm 20.0$ to $15.1 \pm 15.1 \mathrm{ml}, P<0.0001$ ), and it decreased further in 14-day result $(6.9 \pm 10.4 \mathrm{ml}$, $P<0.0001)$. Patients' neurological function was improved remarkably with NIHSS scores from baseline 13 points to 7 -day 7 points $(P<0.0001)$ and 14 -day 4 points $(P<0.0001)$. Cerebral edema did not change a lot after 7-day Naoxueshu treatment but it was relieved significantly 14 days after receiving treatments (from 3 to 2 points, $P<0.0001$ ).

Safety outcomes are showed in Table 3. Seven days after Naoxueshu and other treatment, patients' thrombin time (TT) decreased by $0.6 \mathrm{~s}$ (95\% CI: $-1.2 \sim-0.3$, $P=0.036)$, fibrinogen (Fib) increased by $0.8 \mathrm{~g} / 1$ (95\% CI: $0.4 \sim 1.1, P<0.0001$ ), and alanine aminotransferase (ALT) increased by $22.2 \mathrm{U} / 1$ (95\% CI: 7.0 37.5, $P=0.002)$. These changes were not significant in 14-day result. On the other hand, hemoglobin $(\mathrm{Hgb})$ decreased by $5.7 \mathrm{~g} / \mathrm{l}(95 \%$ CI: $-10.9 \sim-0.5, P=0.028$ ) and platelet (PLT) increased by $41.0 \times 10^{9} / 1(95 \% \mathrm{CI}: 13.7 \sim 68.3, P=0.002) 14$ days after treatment.

\section{Linear regression of risk factors}

Factors related to hematoma volume Male sex was related independently to small 7-day hematoma volume $(B=-8.5$, $95 \% \mathrm{CI}:-15.5 \sim-1.5, P=0.019$, Table 4$)$. If SICH was not caused by hypertension or intracranial vessel malformation, it was more likely linking to a large 7-day hematoma volume ( $B=20.4,95 \%$ CI: $2.0 \sim 38.8, P=0.031$, Table 4). Patients without craniocerebral surgery seemed to have higher risk of large 7-day hematoma volume $(B=8.6,95 \% \mathrm{CI}$ : $0.2 \sim 17.0$, $P=0.045$, Table 4). Not surprisingly, large baseline hematoma volume was related to large 7-day hematoma volume $(B=0.3,95 \%$ CI: $0.03 \sim 0.6, P=0.030$, Table 4$)$. However, only rare cause of SICH (not hypertensive or caused by intracranial vessel malformation) was related to large 14-day hematoma volume $(B=17.4,95 \%$ CI: 4.6 30.1, $P=0.009$, Table 4).
Table 3 Safety outcome measures of the patients

\begin{tabular}{lcccc}
\hline Outcomes & Baseline & 7-day & 14-day & $P$ value \\
\hline WBC $\left(\times 10^{9} / \mathrm{l}\right)$ & $10.5 \pm 4.5$ & $9.2 \pm 3.0$ & $8.0 \pm 3.2$ & 0.013 \\
Hgb $(\mathrm{g} / \mathrm{l})$ & $137.0 \pm 19.2$ & $130.9 \pm 22.3$ & $130.1 \pm 18.5$ & 0.002 \\
PLT $\left(\times 10^{9} / \mathrm{l}\right)$ & $200.4 \pm 73.7$ & $228.3 \pm 76.6$ & $244.2 \pm 83.9$ & $<0.0001$ \\
APTT $(\mathrm{s})$ & $29.2 \pm 6.6$ & $29.2 \pm 6.5$ & $30.3 \pm 5.9$ & 0.269 \\
PT $(\mathrm{s})$ & $12.5 \pm 2.4$ & $12.3 \pm 2.0$ & $12.2 \pm 1.8$ & 0.211 \\
TT $(\mathrm{s})$ & $16.7 \pm 3.0$ & $16.1 \pm 2.9$ & $16.0 \pm 2.7$ & 0.012 \\
Fib $(\mathrm{g} / \mathrm{l})$ & $3.1 \pm 1.1$ & $3.8 \pm 1.6$ & $3.7 \pm 1.4$ & $<0.0001$ \\
ALT $(\mathrm{U} / \mathrm{l})$ & $26.2 \pm 15.9$ & $43.7 \pm 39.2$ & $40.4 \pm 39.9$ & $<0.0001$ \\
AST $(\mathrm{U} / \mathrm{l})$ & $28.1 \pm 16.2$ & $39.8 \pm 58.6$ & $35.5 \pm 37.6$ & 0.034 \\
TBIL $(\mu \mathrm{mol} / \mathrm{l})$ & $17.9 \pm 8.5$ & $16.7 \pm 11.5$ & $13.5 \pm 7.8$ & 0.018 \\
Cr $(\mu \mathrm{mol} / \mathrm{l})$ & $64.2 \pm 24.6$ & $62.1 \pm 21.2$ & $63.1 \pm 32.4$ & 0.570 \\
BUN $(\mathrm{mmol} / \mathrm{l})$ & $6.3 \pm 7.3$ & $8.8 \pm 10.5$ & $8.7 \pm 14.7$ & 0.902 \\
\hline
\end{tabular}

Notes: $W B C$, white blood cell; $H g b$, hemoglobin; PLT, platelet; $A P T T$, activated partial thromboplastin time; $P T$, prothrombin time; $T T$, thrombin time; Fib, fibrinogen; $A L T$, alanine aminotransferase; $A S T$, aspartate aminotransferase; $T B I L$, total bilirubin; $C r$, creatinine; $B U N$, blood urea nitrogen

Factors related to neurological function Again, male sex was related independently to better 7-day NIHSS score $(B=-4.4,95 \% \mathrm{CI}:-7.6 \sim-1.25, P=0.009$, Table 5). Patients' respiratory rate $(B=0.7,95 \% \mathrm{CI}: 0.1 \sim 1.3$, $P=0.032)$ and body temperature $(B=-2.2,95 \%$ CI: $-4.4 \sim-0.1, P=0.039$, Table 5) at the time when SICH symptom onset could also affect their 7-day NIHSS score. Apparently, worse baseline NIHSS score was related to worse 7-day NIHSS score $(B=0.5,95 \% \mathrm{CI}: 0.4 \sim 0.7$, $P<0.0001)$ and 14 -day NIHSS score $(B=0.3,95 \% \mathrm{CI}$ : $0.1 \sim 0.5, P=0.003$, Table 5). Interestingly, immediate use of Naoxueshu seemed to have worse 7-day $(B=-3.4,95 \%$ CI: $-5.1 \sim-1.7, P<0.0001)$ and 14-day NIHSS score $(B=-2.9,95 \%$ CI: $-4.9 \sim-1.0, P=0.005$, Table 5$)$.

No statistically significant risk factor was found that was related to cerebral edema (Table 6).

\section{Discussion}

The results of our study show that Naoxueshu oral liquid is an effective and safe treatment in reducing hematoma volume and cerebral edema in SICH.
Table 2 Efficacy outcome measures of the patients

\begin{tabular}{lllll}
\hline Outcomes & Baseline & 7-day & 14-day & $P$ value \\
\hline Hematoma volume (ml) & $27.3 \pm 20.0$ & $15.1 \pm 15.1$ & $6.9 \pm 10.4$ & $<0.0001$ \\
NIHSS score (median, IQR) & $13(4-20)$ & $7(2-13)$ & $4(0-10)$ & $<0.0001$ \\
Cerebral edema score (median, IQR) & $3(2-3)$ & $2(2-3)$ & $2(1-2)$ & $<0.0001$ \\
\hline
\end{tabular}

Notes: $I Q R$, interquartile range; NIHSS, National Institutes of Health Stroke Scale 
Table 4 Factors related to hematoma volume
Table 5 Factors related to neurological function

\begin{tabular}{|c|c|c|c|c|}
\hline \multirow[t]{2}{*}{ Factors } & \multicolumn{2}{|l|}{ 7-day hematoma volume } & \multicolumn{2}{|c|}{ 14-day hematoma volume } \\
\hline & $B(95 \% \mathrm{CI})$ & $P$ value & $B(95 \% \mathrm{CI})$ & $P$ value \\
\hline Age & $0.01(-0.3 \sim 0.3)$ & 0.963 & $0.04(-0.2 \sim 0.2)$ & 0.673 \\
\hline Male sex & $-8.5(-15.5 \sim-1.5)$ & 0.019 & $-1.7(-6.6 \sim 3.1)$ & 0.468 \\
\hline Heart rate & $0.05(-0.2 \sim 0.2)$ & 0.623 & $-0.04(-0.2 \sim 0.1)$ & 0.580 \\
\hline Respiratory rate & $0.4(-1.0 \sim 1.8)$ & 0.572 & $0.7(-0.3 \sim 1.6)$ & 0.167 \\
\hline Temperature & $-2.4(-7.0 \sim 2.2)$ & 0.301 & $-1.1(-4.3 \sim 2.1)$ & 0.486 \\
\hline Systolic pressure & $0.07(-0.06 \sim 0.2)$ & 0.270 & $0.04(-0.05 \sim 0.1)$ & 0.370 \\
\hline Diastolic pressure & $-0.08(-0.3 \sim 0.1)$ & 0.422 & $0.02(-0.1 \sim 0.2)$ & 0.765 \\
\hline \multicolumn{5}{|l|}{ Cause of SICH } \\
\hline Hypertension & l & l & l & l \\
\hline Intracranial vessel malformation & $0.1(-7.4 \sim 7.6)$ & 0.970 & $2.9(-2.3 \sim 8.1)$ & 0.262 \\
\hline Others & $20.4(2.0 \sim 38.8)$ & 0.031 & $17.4(4.6 \sim 30.1)$ & 0.009 \\
\hline Baseline hematoma volume & $0.3(0.03 \sim 0.6)$ & 0.030 & $0.09(-0.1 \sim 0.3)$ & 0.382 \\
\hline Baseline NIHSS score & $0.002(-0.4 \sim 0.4)$ & 0.991 & $-0.05(-0.2 \sim 0.3)$ & 0.695 \\
\hline Baseline cerebral edema score & $-1.1(-4.1 \sim 1.9)$ & 0.475 & $-1.3(-3.4 \sim 0.8)$ & 0.209 \\
\hline \multicolumn{5}{|l|}{ Surgery } \\
\hline Yes & l & l & l & l \\
\hline No & $8.6(0.2 \sim 17.0)$ & 0.045 & $3.0(-2.8 \sim 8.8)$ & 0.297 \\
\hline Time from onset to receive NXS & $-0.1(-3.7 \sim-3.5)$ & 0.960 & $-0.6(-3.1 \sim 1.9)$ & 0.652 \\
\hline
\end{tabular}

Notes: SICH, spontaneous intracerebral hemorrhage; NIHSS, National Institutes of Health Stroke Scale; $N X S$, Naoxueshu oral liquid

\begin{tabular}{|c|c|c|c|c|}
\hline \multirow[t]{2}{*}{ Factors } & \multicolumn{2}{|l|}{ 7-day NIHSS score } & \multicolumn{2}{|l|}{ 14-day NIHSS score } \\
\hline & $B(95 \%$ CI $)$ & $P$ value & $B(95 \% \mathrm{CI})$ & $P$ value \\
\hline Age & $0.02(-0.1 \sim 0.2)$ & 0.702 & $0.02(-0.1 \sim 0.2)$ & 0.762 \\
\hline Male sex & $-4.4(-7.6 \sim-1.2)$ & 0.009 & $-3.4(-7.2 \sim 0.4)$ & 0.079 \\
\hline Heart rate & $0.1(-0.02 \sim 0.3)$ & 0.103 & $0.1(-0.04 \sim 0.2)$ & 0.229 \\
\hline Respiratory rate & $0.7(0.1 \sim 1.3)$ & 0.032 & $0.4(-0.4 \sim 1.1)$ & 0.331 \\
\hline Temperature & $-2.2(-4.4 \sim-0.1)$ & 0.039 & $-2.2(-4.8 \sim 0.3)$ & 0.079 \\
\hline Systolic pressure & $0.02(-0.04 \sim 0.1)$ & 0.471 & $0.01(-0.1 \sim 0.1)$ & 0.776 \\
\hline Diastolic pressure & $-0.04(-0.1 \sim 0.1)$ & 0.409 & $-0.1(-0.2 \sim 0.1)$ & 0.379 \\
\hline \multicolumn{5}{|l|}{ Cause of SICH } \\
\hline Hypertension & / & l & l & l \\
\hline Intracranial vessel malformation & $-0.06(-3.4 \sim 3.5)$ & 0.973 & $0.7(-3.4 \sim 4.8)$ & 0.721 \\
\hline Others & $6.8(-1.6 \sim 15.3)$ & 0.110 & $3.6(-6.5 \sim 13.7)$ & 0.472 \\
\hline Baseline hematoma volume & $0.02(-0.1 \sim 0.2)$ & 0.750 & $-0.03(-0.2 \sim 0.1)$ & 0.721 \\
\hline Baseline NIHSS score & $0.5(0.4 \sim 0.7)$ & $<0.0001$ & $0.3(0.1 \sim 0.5)$ & 0.003 \\
\hline Baseline cerebral edema score & $-0.2(-1.6 \sim 1.2)$ & 0.774 & $0.7(-1.0 \sim 2.3)$ & 0.406 \\
\hline \multicolumn{5}{|l|}{ Surgery } \\
\hline Yes & / & / & l & l \\
\hline No & $-1.5(-5.3 \sim 2.4)$ & 0.443 & $-2.7(-7.3 \sim 1.9)$ & 0.235 \\
\hline Time from onset to receive NXS & $-3.4(-5.1 \sim-1.7)$ & $<0.0001$ & $-2.9(-4.9 \sim-1.0)$ & 0.005 \\
\hline
\end{tabular}

Notes: SICH, spontaneous intracerebral hemorrhage; NIHSS, National Institutes of Health Stroke Scale; $N X S$, Naoxueshu oral liquid
Use of Naoxueshu oral liquid in stroke treatment can be dated back to the year 2000. Nearly two decades later, in 2017, a systematic review and meta-analysis of 14 studies
(1606 patients) was conducted and revealed the efficacy of Naoxueshu in SICH [15]. However, high heterogeneity was found in some of their results, and the authors explained that 
Table 6 Factors related to cerebral edema

\begin{tabular}{|c|c|c|c|c|}
\hline \multirow[t]{2}{*}{ Factors } & \multicolumn{2}{|c|}{ 7-day cerebral edema score } & \multicolumn{2}{|c|}{ 14-day cerebral edema score } \\
\hline & $B(95 \% \mathrm{CI})$ & $P$ value & $B(95 \% \mathrm{CI})$ & $P$ value \\
\hline Age & $-0.01(-0.05 \sim 0.03)$ & 0.519 & $-0.003(-0.04 \sim 0.04)$ & 0.876 \\
\hline Male sex & $-0.2(-0.8 \sim 1.1)$ & 0.728 & $0.2(-0.7 \sim 1.2)$ & 0.608 \\
\hline Heart rate & $-0.02(-0.05 \sim 0.006)$ & 0.121 & $-0.01(-0.04 \sim 0.02)$ & 0.358 \\
\hline Respiratory rate & $0.08(-0.1 \sim 0.3)$ & 0.398 & $-0.01(-0.2 \sim 0.2)$ & 0.891 \\
\hline Temperature & $0.3(-0.4 \sim 0.9)$ & 0.406 & $-0.1(-0.8 \sim 0.5)$ & 0.637 \\
\hline Systolic pressure & $0.0001(-0.02 \sim 0.02)$ & 0.959 & $-0.005(-0.02 \sim 0.01)$ & 0.581 \\
\hline Diastolic pressure & $0.01(-0.01 \sim 0.05)$ & 0.277 & $0.02(-0.01 \sim 0.05)$ & 0.160 \\
\hline \multicolumn{5}{|l|}{ Cause of SICH } \\
\hline Hypertension & l & l & l & / \\
\hline Intracranial vessel malformation & $0.09(-0.9 \sim 1.1)$ & 0.856 & $-0.5(-1.5 \sim 0.6)$ & 0.366 \\
\hline Others & $1.1(-1.4 \sim 3.7)$ & 0.367 & $1.5(-1.1 \sim 4.0)$ & 0.246 \\
\hline Baseline hematoma volume & $-0.01(-0.05 \sim 0.03)$ & 0.739 & $-0.003(-0.04 \sim 0.04)$ & 0.885 \\
\hline Baseline NIHSS score & $-0.01(-0.06 \sim 0.05)$ & 0.847 & $0.007(-0.04 \sim 0.06)$ & 0.779 \\
\hline Baseline cerebral edema score & $0.3(-0.1 \sim 0.8)$ & 0.109 & $0.3(-0.1 \sim 0.7)$ & 0.139 \\
\hline \multicolumn{5}{|l|}{ Surgery } \\
\hline Yes & l & / & / & / \\
\hline No & $-0.2(-1.4 \sim 1.0)$ & 0.737 & $-0.4(-1.5 \sim 0.8)$ & 0.508 \\
\hline Time from onset to receive NXS & $0.1(-0.4 \sim 0.6)$ & 0.590 & $-0.06(-0.6 \sim 0.4)$ & 0.802 \\
\hline
\end{tabular}

Notes: SICH, spontaneous intracerebral hemorrhage; NIHSS, National Institutes of Health Stroke Scale; $N X S$, Naoxueshu oral liquid the reasons might be related to the number of samples, the quality of the literature, the subject of study, and the duration of treatment. In our study, we controlled Naoxueshu taking time (14 days) in a relatively large-scale population (220 subjects) and confirmed the result that Naoxueshu could obviously promote the absorption of intracranial hematoma; promote the recovery of neurological function, effectively reduce brain edema; and improve the prognosis of patients. Cerebral edema after brain injury was considered dangerous because of the following high intracranial pressure and cerebral hernia. Medical treatment for cerebral edema was restricted to osmolar agents such as mannitol and hypertonic saline, and corticosteroids [16]. However, they had limited efficacy with a relatively high potential of risk. Numerous neuroprotective agents had been tested in preclinical models or small clinical trials in patients. To our knowledge, the results were disappointing. Studies demonstrated that components in Naoxueshu oral liquid could reduce inflammatory response and protect blood-brain barrier (BBB) from disruption $[17,18]$. Our study found that Naoxueshu treatment could gradually relieve cerebral edema after $\mathrm{SICH}$ (from baseline 3 points to 14 -day 2 points, $P<0.0001$ ) and it might have potential ability to prevent late cerebral edema.

Although TCM is very popular in Asian countries, safety is the main concern when it is practiced in western countries. Because the active components of TCM often have not been specified and measured precisely, TCM may have direct toxic to the liver or kidney. Moreover, allergic reactions and interactions with other medicines in TCM use can also be seen in some reports. Our study recorded changes in patients' blood routine examination, coagulation function, liver function, and renal function. Gradually increase in PLT (from baseline $200.4 \times 109 / 1$ to 14 -day $244.2 \times 109 / 1$, $P=0.002$ ), which indicated recovery from bleeding, was found. In addition, we also found the level of fib and ALT were elevated 7 days after Naoxueshu treatment. This might be caused by the release of thrombin combined with fibrin secondarily after cerebral hemorrhage, in order to block the ruptured blood vessels and prevent rebleeding. The elevated thrombin and fibrin would cause the increase of ALT and AST levels. This increase was short term and it began to decrease in 14-day results. Changes of $\mathrm{Hgb}$ might be related to loss of blood and changes of TT were considered not clinically significant.

Up till now, over 10 clinical grading scores for $\mathrm{ICH}$ have been developed in order to assist its prognostication [19-26]. The ICH score revealed that factors independently associated with 30-day mortality were Glasgow Coma Scale (GCS) score, age $\geq 80$ years, infratentorial origin of $\mathrm{ICH}$, $\mathrm{ICH}$ volume, and presence of intraventricular hemorrhage [19]. The ICH-GS confirmed the predictors but with different cutoffs and scoring to improve the prognostic power of the predictors [23]. Another important grading scale for ICH prognosis prediction was the FUNC score, which could accurately identify patients with low chance of functional neurologic recovery at discharge. Related predictive factors 
were age, GCS, ICH location, volume, and pre-ICH cognitive impairment [24]. In our study, besides high baseline NIHSS score, female patients with high respiratory rate and low body temperature might lead to unfavorable 7-day neurologic recovery. However, these factors did not have an impact on 14-day neurological function. Importantly, immediate use of Naoxueshu was associated to worse 7-day and 14-day NIHSS score. There are many factors that affect the deterioration of neurological function in the early stage of cerebral hemorrhage, such as fluctuations in blood pressure, the influence of surgery, and poor control of cerebral edema, so it seems the early neurological function deterioration of cerebral hemorrhage, but it still needs to be comprehensively considered, and pay more attention to the final improvement of neurological function. However, we still consider a relatively later use of Naoxueshu after SICH onset in practice.

Hypertension and intracranial vessel malformation were two major causes of SICH. According to our study, $69.5 \%$ of all the SICH patients were hypertensive, which was higher than the results reported by Finland [27] and the USA [28]. Intracranial vessel malformation led to $27.7 \%$ of SICH, and it was in accordance with the result from another study in Chinese population [29]. Other rare cause of SICH could be internal disease, hematological disease, brain tumor, and some unidentified causes. Although this rare cause only accounted for $2.7 \%$ of all SICH, it was independently related to 7-day $(B=20.4, P=0.031)$ and 14-day hematoma volume $(B=17.4, P=0.009)$. Moreover, surgery relieved hematoma volume temporarily because 14 -day result was not statistically significant. That supported the fact that efficacy of surgical removal of the hemorrhage was still "unclear" [30].

Besides the risk factors discussed above, studies also revealed that homogeneous and regularly shaped small $\mathrm{ICH}$, or benign ICH, and intraventricular hemorrhage (IVH) growth defined as any newly occurring intraventricular bleeding in patients without baseline IVH or an increase in IVH volume $\geq 1 \mathrm{ml}$ in patients with initial IVH on follow-up CT scan within $36 \mathrm{~h}$ after the baseline CT scan were useful risk prediction predictors for outcome in $\mathrm{ICH}$ patients [31, 32]. Moreover, although in our study patients' blood pressure was not a risk factor related to hematoma volume or cerebral edema, there was an opinion that ultra-early blood pressure reduction within $2 \mathrm{~h}$ after onset of symptoms could be an optimal treatment strategy for ICH [33].

The main limitation of our study was that we used a 4-grade scale to evaluate cerebral edema. It was easy to operate in all the 6 hospitals but it was not so accurate. This might affect the results of liner regression in cerebral edema analysis. Second, there is no control group in this observational study, so natural course of hematoma absorption with the increase of time after ICH onset could not be evaluated and analyzed. For the BMI and blood lipid parameters which were also very important to evaluate the outcomes, we did not collect previously, which may have some impact on our conclusion.

\section{Conclusion}

Naoxueshu oral liquid could relieve hematoma volume and cerebral edema safely; meanwhile it could improve patients' neurological function. Sex, cause of SICH, and time from onset to receive Naoxueshu should be taken into consideration in the treatment of SICH. Female patients with SICH that was not caused by hypertension or intracranial vessel malformation should be treated carefully with relatively later use of Naoxueshu oral liquid.

Author contribution JS, YN, and LG designed the study, YN, PW, XQ, and HL performed data collection and statistical analysis, JS wrote the manuscript, and LG revised the manuscript.

Funding This study was supported by the Key Special Project of Ministry of Science and Technology Research on modernization of Traditional Chinese Medicine (2019YFC1712400) and Beijing Traditional Chinese Medicine Science and Technology Development Fund (JJ2020-21) and also was funded by Establishment of National Prominent Traditional Chinese Medicine Experts Gaoli Inheritance Workstation Project (Grant/Award Number:[2016] 42).

Data availability The original data will be available when contacting the correspondence author.

\section{Declarations}

Ethical approval None.

Conflict of interest The authors declare no competing interests.

Open Access This article is licensed under a Creative Commons Attribution 4.0 International License, which permits use, sharing, adaptation, distribution and reproduction in any medium or format, as long as you give appropriate credit to the original author(s) and the source, provide a link to the Creative Commons licence, and indicate if changes were made. The images or other third party material in this article are included in the article's Creative Commons licence, unless indicated otherwise in a credit line to the material. If material is not included in the article's Creative Commons licence and your intended use is not permitted by statutory regulation or exceeds the permitted use, you will need to obtain permission directly from the copyright holder. To view a copy of this licence, visit http://creativecommons.org/licenses/by/4.0/.

\section{References}

1. Woo D, Broderick JP (2002) Spontaneous intracerebral hemorrhage: epidemiology and clinical presentation. Neurosurg Clin $\mathrm{N}$ Am 13:265-279

2. National Institute of Neurological Disorders and Stroke (1990) Classification of cerebrovascular diseases III. Stroke 21:647-676 
3. Broderick JP, Brott TG, Duldner JE et al (1993) Volume of intracerebral hemorrhage a powerful and easy-to-use predictor of 30-day mortality. Stroke 24:987-993

4. Hemphill JC, Bonovich DC, Besmertis L et al (2001) The ICH score: a simple, reliable grading scale for intracerebral hemorrhage. Stroke 32:891-897

5. Hemphill JC 3rd, Greenberg SM, Anderson CS et al (2015) Guidelines for the management of spontaneous intracerebral hemorrhage: a guideline for healthcare professionals from the American Heart Association/American Stroke Association. Stroke 46:2032-2060

6. Chamorro Á, Dirnagl U, Urra X, Planas AM (2016) Neuroprotection in acute stroke: targeting excitotoxicity, oxidative and nitrosative stress, and inflammation. Lancet Neurol 15:869-881

7. World Health Organization. World Health Organization Fact Sheet. Revised May 2003 ed. Geneva: World Health Organization; 2003.

8. Chen C, Yuan LX, Zhang GM (2014) Clinical application and experimental research progress of nao-xue-shu oral liquid in cerebrovascular disease. Chinese Journal of Integrated Traditional Chinese and Western Medicine 12:1005-1006

9. Wang XG, Zhao XQ (2015) Paired study on the treatment of acute cerebral hemorrhage with nao-xue-shu oral liquid. Chinese Journal of Integrative Medicine on Cardio-/Cerebrovascular Disease 13:937-938

10. Chen SJ, Wang HY, Zuo Y et al (2016) Clinical observation on the treatment of secondary brain damage after intracerebral hemorrhage by Nao-Xue-Shu oral liquid. Chin J Integr Med 14:199-202

11. Miao WC, Yan R (2014) Clinical observation on the treatment of hypertensive cerebral hemorrhage with naoxueshu oral liquid. Chinese Journal of Practical Nervous Disease 17:102-103

12. Wang H, Niu XZ, Su TS (2016) Clinical observation on the treatment of cerebral hemorrhage in convalescent stage with acupuncture combined with naoxueshu oral liquid. World Latest Medicine Information 16:110-111

13. Li SH, Han XH, Jiang GY et al (2014) Clinical observation on the treatment of hypertensive cerebral hemorrhage with minimally invasive surgery of intracranial hematoma combined with naoxueshu oral liquid. Chinese Journal of Practical Nervous Diseases $17: 82-83$

14. Song J, Lyu Y, Wang P et al (2018) Treatment of Naoxueshu promotes improvement of hematoma absorption and neurological function in acute intracerebral hemorrhage patients. Front Physiol 9:933

15. Wu L, Li Y, Wang X et al (2017) A systematic review and metaanalysis on the treatment of cerebral hemorrhage with NaoXueShu oral liquid. Biomed Res Int 2017:8542576

16. Kim H, Edwards NJ, Choi HA et al (2016) Treatment strategies to attenuate perihematomal edema in patients with intracerebral hemorrhage. World Neurosurg 94:32-41

17. Dong H, Ren JX, Wang JJ et al (2016) Chinese medicinal leech: ethnopharmacology, phytochemistry, and pharmacological activities. Evid Based Complement Alternat Med 2016:7895935

18. Li KM, Zhang G, Wu JB (2007) Overview of pharmacological research of leech. Tradit Chin Med Res 20:62-64
19. Hemphill JC, Bonovich DC, Besmertis L et al (2011) The ICH score: a simple, reliable grading scale for intracerebral hemorrhage. Stroke 32:891-897

20. Cheung RT, Zou LY (2003) Use of the original, modified, or new intracerebral hemorrhage score to predict mortality and morbidity after intracerebral hemorrhage. Stroke 34:1717-1722

21. Godoy DA, Pinero G, Di Napoli M (2006) Predicting mortality in spontaneous intracerebral hemorrhage: can modification to original score improve the prediction? Stroke 37:1038-1044

22. Weimar C, Benemann J, Diener HC (2006) Development and validation of the Essen intracerebral haemorrhage score. J Neurol Neurosurg Psychiatry 77:601-605

23. Ruiz-Sandoval JL, Chiquete E, Romero-Varguss S et al (2007) Grading scale for prediction of outcome in primary intracerebral hemorrhages. Stroke 38:1641-1644

24. Rost NS, Smith EE, Chang Y et al (2008) Prediction of functional outcome in patients with primary intracerebral hemorrhage: the FUNC score. Stroke 39:2304-2309

25. Cho DY, Chen CC, Lee WY et al (2008) A new modified intracerebral hemorrhage score for treatment decision in basal ganglia hemorrhage: a randomized trial. Crit Care Med 36:2151-2156

26. Chaung YC, Chen YM, Peng SK, Peng SY (2009) Risk stratification for predicting 30-day mortality of intracerebral hemorrhage. Int J Qual Health Care 21:441-447

27. Fogelholm R, Murros K (1993) Cigarette smoking and risk of primary intracerebral haemorrhage. A population-based case-control study. Acta Neurol Scand 87:367-370

28. Feldmann E, Broderick JP, Kernan WN et al (2005) Major risk factors for intracerebral hemorrhage in the young are modifiable. Stroke 36:1881-1885

29. Luo J, Wang YH, Zhong XN et al (2008) Analysis of etiological factor, risk factor and prognosis of 158 young patients with intracerebral hemorrhage. Modern Preventive Medicine 35:1587-1589

30. Manners J, Steinberg A, Shutter L (2017) Early management of acute cerebrovascular accident. Curr Opin Crit Care 23:556-560

31. Li Q, Yang WS, Shen YQ et al (2019) Benign intracerebral hemorrhage: a population at low risk for hematoma growth and poor outcome. J Am Heart Assoc 8(8):e011892

32. Li Q, Li R, Zhao LB et al (2020) Intraventricular hemorrhage growth: definition, prevalence and association with hematoma expansion and prognosis. Neurocrit Care 33(3):732-739. https:// doi.org/10.1007/s12028-020-00958-8

33. Li Q, Warren AD, Qureshi AI et al (2020) Ultra-early blood pressure reduction attenuates hematoma growth and improves outcome in intracerebral hemorrhage. Ann Neurol 88(2):388-395. https://doi.org/10.1002/ana.25793

Publisher's note Springer Nature remains neutral with regard to jurisdictional claims in published maps and institutional affiliations. 